\title{
A narrative review of research progress on the relationship between hypoxia-inducible factor- $2 \alpha$ and wound angiogenesis
}

\author{
Xing-Wang Xu, Jie Zhang, Zai-Wen Guo, Ming-Ming Song, Ran Sun, Xin-Yuan Jin, Jian-Dong Su, \\ Bing-Wei Sun
}

Department of Burns and Plastic, Affiliated Suzhou Hospital of Nanjing Medical University, Suzhou, China

Contributions: (I) Conception and design: XW Xu, J Zhang; (II) Administrative support: JD Su; (III) Provision of study materials or patients: ZW Guo; (IV) Collection and assembly of data: MM Song; (V) Data analysis and interpretation: R Sun; (VI) Manuscript writing: All authors; (VII) Final approval of manuscript: All authors.

Correspondence to: Jian-Dong Su, MD; Bing-Wei Sun, MD. Department of Burns and Plastic, Affiliated Suzhou Hospital of Nanjing Medical University, 242 Guangji Road, Suzhou 215008, China. Email: jiandongsu@njmu.edu.cn; sunbinwe@hotmail.com.

\begin{abstract}
Objective: This article aims to pay attention to the latest research on the expression, activation and function of hypoxia-inducible factor- $2 \alpha$ (HIF-2 $\alpha$ ) under hypoxia and non-hypoxia conditions, and summarizes the current knowledge about the interaction between hypoxia-inducible factor- 2 and angiogenesis, hoping to understand its actions in physiology and disease, with the goal of providing a new strategy for the diagnosis and treatment of wounds.

Background: Wound healing is a complex and continuous process, involving coagulation, inflammation, angiogenesis, new tissue formation and extracellular matrix remodeling. Of these, angiogenesis is an essential step. One of the main reasons for non-healing or delayed healing of wounds in peripheral vascular diseases and diabetes is the reduced ability to regenerate microvessels through the process of angiogenesis, which has become the focus of new methods for treating chronic wounds. HIF-2 $\alpha$ regulates many aspects of angiogenesis, including vascular maturation, cell migration, proliferation and metastasis.

Methods: Throughout extensive search of PubMed, summarize the medical research on HIF-2 $\alpha$ to 2020.

Conclusions: HIF-2 $\alpha$ is necessary for normal embryonic development by stimulating the expression of angiogenic factors, such as vascular endothelial growth factor (VEGF). It is essential for the formation of new blood vessels in physiological and pathophysiological environments. Targeting HIF-2 $\alpha$ in wound healing has much clinical significance for tissue repair.
\end{abstract}

Keywords: Angiogenesis; wound healing; chronic wounds; hypoxia-inducible factor-2 $\alpha$ (HIF-2 $\alpha$ )

Submitted Jan 27, 2021. Accepted for publication Apr 21, 2021.

doi: 10.21037/apm-21-450

View this article at: http://dx.doi.org/10.21037/apm-21-450

\section{Introduction}

Wound repair is a complex process that requires the cooperation of many types of cells and microenvironments. An important feature of wound healing is the apparent increase in microvessel density, known as angiogenesis (1). The formation of microvessels will bring oxygen, immune cells and nutrients to assist in wound healing. Hypoxiainducible factor $2 \alpha$ (HIF-2 $\alpha$ ) upregulates the genes that encode almost every step of angiogenesis, including the initiation, formation and maturation of blood vessels. Therefore, the overall goal of our work is to summarize the current progress on the relationship between HIF- $2 \alpha$ and microvascular generation.

We present the following article in accordance with the Narrative Review reporting checklist (available at http:// dx.doi.org/10.21037/apm-21-450). 


\section{Methods}

We use the PubMed to retrieve for clinical studies which involves prospective and retrospective studies regarding HIF-2 $\alpha$. The publication date of searched literatures was from the inception of databases to 2021 and there were no restrictions on publication regions, languages or types. The following MeSH terms and their combinations were used in[Tittle/abstract]: "Hypoxia-inducible factor $2 \alpha$ " OR "Angiogenesis" OR “target genes" OR “clinical trial” OR "chronic wound".

\section{Structure and function of HIF}

HIF is considered to be the most important regulator of the cellular response to hypoxia, and has the function of regulating the expression of genes related to hypoxic stress in tissue cells. In 1993, Semenza and Wang discovered HIF in their study of erythropoietin (EPO) (2). There are three subtypes of HIF, HIF-1, HIF-2 and HIF-3, and they are respectively composed of sequence homologous HIF- $1 \alpha$ (gene coding HIF-1A), HIF- $2 \alpha$ (gene coding EPAS1), HIF$3 \alpha$ (gene coding HIF-3A) and HIF- $\beta$. The dimer consists of an oxygen-sensitive $\alpha$-subunit and a stable $\beta$-subunit that is not regulated by oxygen concentration. The $\alpha$-subunit consists of the following structures: the amino terminal bHLH for DNA binding and the PAS-A and PAS-B domains required for heterodimerization (3). In the middle is the oxygen-dependent degradation domain (ODDD), associated with degradation of the HIF- $\alpha$ subunit. ODDD is a highly conserved domain that controls the activity and stability of the $\alpha$-subunit, because it contains asparagine $(\mathrm{N})$ and proline $(\mathrm{P})$ residues for hydroxylation under normoxic conditions; the terminal of the $\alpha$-subunit is the $\mathrm{C}$-terminal and $\mathrm{N}$-terminal transactivation domains (N-TAD and C-TAD), which are needed to activate HIF target genes. The existence of two different TAD ensures the $\alpha$-subunits have common and unique regulatory properties (4). Under normoxic conditions, proline hydroxylase (PHD) hydroxylates two $\mathrm{P}$ residues of the HIF- $\alpha$ subunit, which requires oxygen $\left(\mathrm{O}_{2}\right)$, iron $[\mathrm{Fe}(2+)]$ and $\alpha$-ketoglutaric acid as co-substrate. After hydroxylation, the HIF- $\alpha$ subunit can interact and bind with von Hippel-Lindau tumor suppressor protein (VHL) and then degrade via the ubiquitin-proteasome pathway (5). In the hypoxic environment, PHD cannot hydroxylate the $\alpha$-subunit and VHL cannot ubiquitinate the substrate. HIF- $\alpha$ translocates into the nucleus and binds to its corresponding heterodimer
HIF- $\beta$ to continuously produce active HIF. Next, the transactivation domain of the HIF- $\alpha$ subunit protein binds to transcriptional coactivator $\mathrm{p} 300$ and CREB binding protein (CBP), and HIF then has transcriptional activity, thus binding with the hypoxia-response element (HRE) to regulate the expression of various target genes (6).

HIF- $1 \alpha$ and HIF- $2 \alpha$ directly or indirectly regulate hundreds of genes and play a role in iron metabolism, erythropoiesis and other physiological processes. They are also related to the occurrence of some diseases. HIF- $2 \alpha$ is mainly distributed in vascular endothelial cells and fetal lung fibroblasts. Through a series of target genes, such as EPO, plasminogen activator inhibitor 1, liver enrichment gene 1, etc., HIF- $2 \alpha$ plays an important role in iron metabolism, erythropoiesis, angiogenesis, hypoxia adaptation, fetal lung maturation, liver growth and so on.

\section{Angiogenesis}

In growth and development of tissue and organ regeneration and many pathological conditions (7), angiogenesis plays an important role. It depends on interactions between endothelial cells, fibroblasts, macrophages and the surrounding extracellular matrix (ECM) (8). Angiogenesis can be divided into stages of rest, activation and regression. The resting period means that in normal, healthy tissue, the microvascular system is in homeostasis, in which blood vessels are fully perfused to deliver sufficient nutrients and oxygen to tissues. In addition to anti-angiogenic factors such as Angiopoietin-1 (Ang-1) and pigment epithelium-derived factor (PEDF), the body also expresses pro-angiogenic factors such as fibroblast growth factor (FGF) and Vascular endothelial growth factor (VEGF) to maintain a functional vascular network that neither proliferates nor weakens (9). When tissue is injured, the local microvascular system is destroyed, leading to local inflammation and hypoxia, which activate endothelial cells, leading to immune cell recruitment. The injured tissue attracts neutrophils in the early stage, followed by the recruitment of macrophages. By quantifying the degree of vascular germination in vitro, Pylaeva et al. found that neutrophils could inhibit the growth of blood vessels, by inhibiting neutrophils, interferon has pro-angiogenic properties (10). Gurevich et al. observed the process of angiogenesis in mice and zebrafish with a realtime imaging system, and concluded that inflammatory macrophages play a special role in initiating and driving vascular germination by releasing VEGF-A. Another role of inflammatory macrophages in promoting angiogenesis 
is to remove inhibitory neutrophils of wound blood vessel sprouting tips (11). In addition, local hypoxia activates the transcription of HIF- $\alpha$, which promotes angiogenesis through a variety of mechanisms, including regulation of many genes and production of growth factors related with angiogenesis, which involve stromal cell-derived factor 1 (SDF-1), Ang-2 and VEGF-A (5). The proliferative phase of wound healing is usually the peak of angiogenesis (12), after which the wound healing process enters the remodeling stage, and the pro-angiogenic media in the wound gradually degrade, while the expression of anti-angiogenic factors, such as PEDF and Sprouty2 protein, increase, resulting in degeneration of the vascular network $(1,13)$.

\section{HIF-2 $\alpha$ and angiogenesis}

Hypoxia not only affects the development of vascular in earliest steps, but also coordinates angiogenesis in adults under physiological and pathological conditions. The main regulators of $\mathrm{O}_{2}$ homeostasis are HIF- $1 \alpha$ and HIF$2 \alpha$. Although their structure, function and regulation are similar, they do have different and non-redundant functions in development. In the process of angiogenesis, most of the research has been on HIF-1 $\alpha$, although increasing research is showing that HIF-2 $\alpha$ is also very important in angiogenesis. Testis-specific gene antigen 10 (TSGA10) is a normal kind of protein which over expresses in most cancers, it has been proved to exert potent inhibitory effects by disrupting HIF- $1 \alpha$ axis. HIF- $2 \alpha$ (EPAS 1 ) is highly homologous to HIF-1 $\alpha$, and has a preferential expression pattern in endothelial cells. Contrary to HIF-1 $\alpha$, EPAS1 is mainly expressed in vascular endothelial cells. According to the research of Amoorahim et al., based on their analysis, the binding affinity of TSGA10 to HIF- $2 \alpha$ was proved (which leads to the inhibition of HIF-2 $\alpha$ ). It is speculated that the overexpression of TSGA10 may lead to the inhibition of angiogenesis by blocking HIF-2 $\alpha$ in endothelial cells (14). By the analysis of cultured HIF-2 $\alpha$-deficient ECs Skuli et al. did, the mechanism of HIF- $2 \alpha$ regulating the process of pathological angiogenesis and revascularization may be related to HIF-2 $\alpha$-dependent expression of specific angiogenic factors, which involve angiopoietin 2, a Notch ligand and delta-like ligand 4 (DIl4) (15). HIF-2 $\alpha$ directly or indirectly regulates the expression of many genes, the induced genes involving angiogenesis are upregulated, such as basic fibroblast growth factor (bFGF) (16), vascular endothelial growth factor A (VEGF-A) (17), and tumor necrosis factor alpha (TNF- $\alpha$ ), platelet-derived growth factor (PDGF) (18), extracellular matrix (ECM) also plays a certain role in angiogenesis, and MMPs is one of the main proteases involved in ECM degradation, HIF-2 $\alpha$ can also promote angiogenesis by regulating the expression of MMPS (19).

\section{HIF-2a knockout models}

HIF-2 $\alpha$ expresses highly in vascular endothelial cells and it can activate the expression of target genes, and its target products can regulate angiogenesis and the function of blood vessel. Peng et al. observed that HIF-2 $\alpha$ mutant mouse embryos showed severe defects in vessel and bleeding in the embryo and the yolk sac, indicating that HIF- $2 \alpha$ is necessary for normal remodeling/maturation after angiogenesis (20). In 2009, Skuli et al. introduced a mouse model with HIF$2 \alpha$-deficient endothelial cells, which showed increased vascular permeability, abnormal ultrastructure of the endothelial cells, pulmonary hypertension, slower tumor growth, decreased angiogenesis, and impaired expression of angiogenic genes encodingangiopoietin-2, kinase insertion domain receptor $(\mathrm{kdr} / \mathrm{flk}-1)$, fms-associated tyrosine kinase-1 (flt-1) and dll4 (21). Martín-Aragón Baudel et al. found that by exogenous administrationoftheHIF- $2 \alpha$ target, EPO, can promote blood vessel generation and nerve regeneration after neonatal ischaemia in rats. Thus, prioritizing the activation of the HIF- $2 \alpha$-dependent arm of hypoxia adaptation may represent a novel strategy to minimize the damage related with ischemic stroke and promote nerve repair and regeneration (22). In 2018, Amin et al. found that HIF- $2 \alpha$ knockout mice died immediately after birth, and the number of endothelial cells decreased. Immunohistochemical results showed that the decrease in the number of endothelial cells weakened the activity of proliferation and angiogenesis, which was the reason for the damage of the alveolar structure in new born mutant mice (23). The factor eukaryotic translation initiation factor 3 subunit e (eIF3e), which is a hypoxia-independent down regulator of HIF-2 $\alpha$, by promoting hypoxia-dependent down-regulation of HIF-2 $\alpha$, the silencing of eIF3e leads to angiogenesis. In 2015, the experimental results of Takuya Hashimoto et al. suggested that in the hindlimb ischemia model of living mouse, a single intramuscular injection of eIF3esiRNA expression plasmid significantly enhanced the perfusion of ischemic injured limbs on the 7th day, which proved that the silencing of eIF3e in mouse primary skeletal myoblasts led to the stability of HIF-2 $\alpha$ and up-regulation 
of angiogenesis and transcription (24). In summary, these results emphasize the important role of HIF- $2 \alpha$ plays in the development of blood vessel, and it is related to the expression of angiogenesis-related genes mediated by HIF- $2 \alpha$.

\section{HIF-2a target genes}

HIF-2 $\alpha$ upregulates the genes that encode nearly all steps of angiogenesis, which involves initiation, angiogenesis as well as maturation. The most important vascular generation factor, VEGF as well as its receptors VEGFR-1, VEGFR-2, are directly induced by HIF- $2 \alpha$ through binding to its HRE under anoxic conditions (25). VEGF is not only the most important specific regulator of endothelial cell growth and differentiation, but also the survival factor of endothelial cells. It exists in several isotypes, which are derived from genetically through selective splicing. VEGF is the most crucial part of angiogenesis, regulating nearly all steps of angiogenesis, involving angiogenic switch, vascular instability, tip cell formation, vascular permeability initiation, and endothelial cell migration and proliferation in the process of neovascularization. Although HIF- $1 \alpha$ as well as HIF-2 $\alpha$ can both induce VEGF family-related genes expressing in the hypoxic tumor microenvironment, many studies have shown that HIF-2 $\alpha$ is more likely to bind to the promoter of VEGF and highly related to the expression of VEGF (26). Other studies have shown that the expression of the VEGF receptor is also significantly increased in hypoxic and ischemic tissues (27).

Recent studies have shown that HIF is also involved in the regulation of Jag2 and Dll4 ligands in the Notch signaling pathway, which plays an important role in tumor angiogenesis (28). The Jag2 ligand is mainly affected by HIF-1 $\alpha$, which increases the extent of neovascularization, whereas HIF-2 $\alpha$ mainly affects the Dll4 ligand. Dll4 prevents excessive sprouting of endothelial cells by inhibiting the expression of VEFGR-2 and the formation of tip cells, while ensuring the formation of the neovascularization lumen, promoting vascular functional maturation, and then remodeling of vascular function (29). In addition to the above mentioned differences in the target genes regulated by HIF-1 and HIF-2, the mechanisms of regulating angiogenesis are also different under different oxygen concentrations (30). Some studies have exposed human pulmonary artery endothelial cells to different oxygen concentrations. The study results revealed that the proliferation of endothelial cells increased in a considerable amount in hypoxia environment, and it was found that the expression of HIF- $1 \alpha$ and HIF- $2 \alpha$ increased at $5 \%$ oxygen concentration, while only the expression of HIF- $2 \alpha$ increased at $10 \%$ oxygen concentration. This shows that in the case of moderate hypoxia, endothelial cell proliferation and angiogenesis are mainly induced by HIF-2 $\alpha$ (31). In addition, HIF- $2 \alpha$ induces the transcription of endothelial nitric oxide synthase in HMEC1 and human umbilical vein endothelial cells under hypoxia by preferentially binding to the HRE in the endothelial nitric oxide synthase promoter (eNOS). Nitric oxide (NO) not only plays an important role in stimulating angiogenesis, but also plays an important role in vasodilation and vascular normalization. Other HIF-2 target genes involved in angiogenesis include the adenosine $\mathrm{A} 2 \mathrm{~A}$ receptor and EPO, which is a vascular generation factor that promotes the proliferation, migration as well as angiogenesis of cell in human pulmonary microvascular endothelial cells (32).

\section{Clinical significance}

In recent years, some new directions have been found for the degradation of HIF- $2 \alpha$ and the decrease in transcriptional activity. HIF- $2 \alpha$ and vascular endothelial growth factor are closely related to the progression of malignant tumors An abundance of tumor-associated macrophages is associated with poor clinical outcome in numerous human cancers, including breast, ovarian, and prostate cancer tumor-associated macrophages contribute to tumor progression, in part, through their section of the potent angiogenic molecule vascular endothelial growth factor (VEGF), which is driven largely by the low $\mathrm{O}_{2}$ concentration within the tumor microenvironment (33). Besides, one of the basic components of inflammation observed in the synovium of the RA joint is the strong proangiogenic drive and the expression of VEGF. This driving force of angiogenesis is clearly responsible for the formation of growing pannus in the joint. Studies have shown that hypoxia is the main cause of angiogenesis in RA, promotes angiogenesis and inflammatory cascade in RA, and leads to the occurrence of RA tendon disease (34). It is possible that hypoxia as well as angiogenesis may also work for RA tendon disease. Deferoxamine (DFO) can inhibit PHD and prevent pyruvate modifying the transcriptional coactivator p300 of HIF- $2 \alpha$, maintaining the transcriptional activity of HIF- $2 \alpha$, promoting the expression of HIF- $2 \alpha$ and angiogenesis, thus accelerating the healing of ischemic chronic wounds (35). Qayoom et al.'s study showed that 
DFO could accelerate wound healing in diabetic rats by increasing neovascularization, reducing inflammation and improving wound maturation. Data showed that when DFO nanoparticles were mixed into Pluronic gel, the protein levels of HIF, transforming growth factor- $\beta$ (TGF- $\beta$ ), VEGF, SDF- $1 \alpha$ and interleukin-10 were conspicuously higher than other factors, and there was more collagen deposition and new angiogenesis in the skin tissue of diabetic rats treated with DFO (36).

\section{Conclusions}

In summary, the HIF pathway is considered to be the main regulator of $\mathrm{O}_{2}$ homeostasis, which controls the expression of many hypoxia target genes, for example, genes involving metabolism, erythropoiesis, angiogenesis, erythropoiesis, cell survival/apoptosis, migration and other tissue-specific functions. In terms of angiogenesis, HIF$2 \alpha$ is necessary for normal embryonic development by stimulating the expression of angiogenic factors, such as VEGF, Ang, VEGFR and integrin. In the physiological and pathophysiological environment, it is essential for the formation of new blood vessels. However, the molecular mechanism of HIF- $2 \alpha$ regulation and its role in angiogenesis are still not comprehensively understood, in-depth study of the mechanism of HIF- $2 \alpha$ in both physiology and angiogenesis can give new direction to wound treatment. Targeting HIF-2 $\alpha$ in wound healing has much clinical significance for tissue repair. To counteract the harmful effects of too much or too little HIF- $2 \alpha$ signal by regulating the expression of HIF- $2 \alpha$ may improve the treatment of wound healing in the future.

\section{Acknowledgments}

Funding: Jiangsu Provincial Health Commission Project (No. H2017071); Suzhou Clinical Medical Center Construction Program (No. SZZXJ201506); Suzhou Medical Key Discipline Project (No. Szxk201811).

\section{Footnote}

Reporting Checklist: The authors have completed the Narrative Review reporting checklist. Available at http:// dx.doi.org/10.21037/apm-21-450

Conflicts of Interest: All authors have completed the ICMJE uniform disclosure form (available at http://dx.doi. org/10.21037/apm-21-450). Dr. JDS reports funding from Jiangsu Provincial Health Commission Project (No. H2017071) and Suzhou Medical Key Discipline Project (No. szxk201811). The other authors have no conflicts of interest to declare.

Ethical Statement: The authors are accountable for all aspects of the work in ensuring that questions related to the accuracy or integrity of any part of the work are appropriately investigated and resolved.

Open Access Statement: This is an Open Access article distributed in accordance with the Creative Commons Attribution-NonCommercial-NoDerivs 4.0 International License (CC BY-NC-ND 4.0), which permits the noncommercial replication and distribution of the article with the strict proviso that no changes or edits are made and the original work is properly cited (including links to both the formal publication through the relevant DOI and the license). See: https://creativecommons.org/licenses/by-nc-nd/4.0/.

\section{References}

1. Michalczyk ER, Chen L, Fine D, et al. Pigment Epithelium-Derived Factor (PEDF) as a Regulator of Wound Angiogenesis. Sci Rep 2018;8:11142.

2. Wang GL, Semenza GL. General involvement of hypoxiainducible factor 1 in transcriptional response to hypoxia. Proc Natl Acad Sci U S A. 1993;90:4304-8.

3. Carmeliet P, Dor Y, Herbert JM, et al. Role of HIF-1alpha in hypoxia-mediated apoptosis, cell proliferation and tumour angiogenesis. Nature 1998;394:485-90. Erratum in: Nature 1998;395:525.

4. Hu CJ, Sataur A, Wang L, et al. The N-terminal transactivation domain confers target gene specificity of hypoxia-inducible factors HIF-1alpha and HIF-2alpha. Mol Biol Cell 2007;18:4528-42.

5. Choudhry H, Harris AL. Advances in Hypoxia-Inducible Factor Biology. Cell Metab 2018;27:281-98.

6. Tirpe AA, Gulei D, Ciortea SM, et al. Hypoxia: Overview on Hypoxia-Mediated Mechanisms with a Focus on the Role of HIF Genes. Int J Mol Sci 2019;20:6140.

7. Johnson KE, Wilgus TA. Vascular Endothelial Growth Factor and Angiogenesis in the Regulation of Cutaneous Wound Repair. Adv Wound Care (New Rochelle) 2014;3:647-61.

8. Morgan C, Nigam Y. Naturally derived factors and their role in the promotion of angiogenesis for the healing of 
chronic wounds. Angiogenesis 2013;16:493-502.

9. Okonkwo UA, DiPietro LA. Diabetes and Wound Angiogenesis. Int J Mol Sci 2017;18:1419.

10. Pylaeva E, Lang S, Jablonska J. The Essential Role of Type I Interferons in Differentiation and Activation of TumorAssociated Neutrophils. Front Immunol 2016;7:629.

11. Gurevich DB, Severn CE, Twomey C, et al. Live imaging of wound angiogenesis reveals macrophage orchestrated vessel sprouting and regression. EMBO J 2018;37:e97786.

12. DiPietro LA. Angiogenesis and wound repair: when enough is enough. J Leukoc Biol 2016;100:979-84.

13. Wietecha MS, Król MJ, Michalczyk ER, et al. Pigment epithelium-derived factor as a multifunctional regulator of wound healing. Am J Physiol Heart Circ Physiol 2015;309:H812-26.

14. Amoorahim M, Valipour E, Hoseinkhani Z, et al. TSGA10 overexpression inhibits angiogenesis of HUVECs: A HIF$2 \alpha$ biased perspective. Microvasc Res 2020;128:103952.

15. Skuli N, Majmundar A, Krock B, et al. Endothelial HIF- $2 \alpha$ regulates murine pathological angiogenesis and revascularization processes. J Clin Invest 2012;122:1427-43.

16. Zhang S, Zhao L, Wang J, et al. HIF-2 $\alpha$ and Oct4 have synergistic effects on survival and myocardial repair of very small embryonic-like mesenchymal stem cells in infarcted hearts. Cell Death Dis 2017;8:e2548.

17. Sun X, Sun B, Babicheva A, et al. Direct Extracellular NAMPT Involvement in Pulmonary Hypertension and Vascular Remodeling. Transcriptional Regulation by SOX and HIF-2 $\alpha$. Am J Respir Cell Mol Biol 2020;63:92-103.

18. Wang P, Meng Q, Wang W, et al. Icariin inhibits the inflammation through down-regulating NF- $\kappa \mathrm{B} /$ HIF-2 $\alpha$ signal pathways in chondrocytes. Biosci Rep 2020;40:BSR20203107.

19. Wang HX, Qin C, Han FY, et al. HIF-2 $\alpha$ as a prognostic marker for breast cancer progression and patient survival. Genet Mol Res 2014;13:2817-26.

20. Peng J, Zhang L, Drysdale L, et al. The transcription factor EPAS-1/hypoxia-inducible factor 2alpha plays an important role in vascular remodeling. Proc Natl Acad Sci U S A 2000;97:8386-91.

21. Skuli N, Liu L, Runge A, et al. Endothelial deletion of hypoxia-inducible factor-2alpha (HIF-2alpha) alters vascular function and tumor angiogenesis. Blood 2009;114:469-77.

22. Martín-Aragón Baudel MA, Rae M, Darlison M, et al. Preferential activation of HIF-2 $\alpha$ adaptive signalling in neuronal-like cells in response to acute hypoxia. PLoS One 2017;12:e0185664.
23. Amin FZ, Yamashita T, Ohneda O. Deterioration of alveolar development in mice with both HIF-3 $\alpha$ knockout and HIF-2 $\alpha$ knockdown. BMC Res Notes 2018;11:449.

24. Hashimoto T, Chen L, Kimura H, et al. Silencing of eIF3e promotes blood perfusion recovery after limb ischemia through stabilization of hypoxia-inducible factor $2 \alpha$ activity. J Vasc Surg 2016;64:219-226.e3.

25. Elvert G, Kappel A, Heidenreich R, et al. Cooperative interaction of hypoxia-inducible factor-2alpha (HIF2alpha) and Ets-1 in the transcriptional activation of vascular endothelial growth factor receptor-2 (Flk-1). J Biol Chem 2003;278:7520-30.

26. Zhao J, Du F, Shen G, et al. The role of hypoxia-inducible factor-2 in digestive system cancers. Cell Death Dis 2015;6:e1600.

27. Figueira RL, Gonçalves FL, Prado AR, et al. Ventilationinduced changes correlate to pulmonary vascular response and VEGF, VEGFR-1/2, and eNOS expression in the rat model of postnatal hypoxia. Braz J Med Biol Res 2018;51:e7169.

28. Zhang P, Yan X, Chen Y, et al. Notch signaling in blood vessels: from morphogenesis to homeostasis. Sci China Life Sci 2014;57:774-80.

29. Lobov I, Mikhailova N. The Role of Dll4/Notch Signaling in Normal and Pathological Ocular Angiogenesis: Dll4 Controls Blood Vessel Sprouting and Vessel Remodeling in Normal and Pathological Conditions. J Ophthalmol 2018;2018:3565292.

30. Loboda A, Jozkowicz A, Dulak J. HIF-1 versus HIF-2-is one more important than the other? Vascul Pharmacol 2012;56:245-51.

31. Loboda A, Jozkowicz A, Dulak J. HIF-1 and HIF-2 transcription factors--similar but not identical. Mol Cells 2010;29:435-42.

32. Geis T, Doring C, Popp R, et al. HIF-2alpha-dependent PAI-1 induction contributes to angiogenesis in hepatocellular carcinoma. Exp Cell Res 2015;331:46-57.

33. Roda JM, Sumner LA, Evans R, et al. Hypoxia-inducible factor- $2 \alpha$ regulates GM-CSF-derived soluble vascular endothelial growth factor receptor 1 production from macrophages and inhibits tumor growth and angiogenesis. J Immunol 2011;187:1970-6.

34. Sivakumar B, Akhavani M, Winlove C, et al. Synovial hypoxia as a cause of tendon rupture in rheumatoid arthritis. J Hand Surg Am 2008;33:49-58.

35. Ding J, Wang X, Chen B, et al. Exosomes Derived from Human Bone Marrow Mesenchymal Stem Cells Stimulated by Deferoxamine Accelerate Cutaneous 
Wound Healing by Promoting Angiogenesis. Biomed Res Int 2019;2019:9742765.

36. Qayoom A, Aneesha V, Anagha S, et al. Lecithin-based deferoxamine nanoparticles accelerated cutaneous wound

Cite this article as: $\mathrm{Xu} X W$, Zhang J, Guo ZW, Song MM, Sun R, Jin XY, Su JD, Sun BW. A narrative review of research progress on the relationship between hypoxia-inducible factor$2 \alpha$ and wound angiogenesis. Ann Palliat Med 2021;10(4):48824888. doi: 10.21037/apm-21-450 healing in diabetic rats. Eur J Pharmacol 2019;858:172478.

(English Language Editor: K. Brown) 